\title{
XMM-Newton observations of the Small Magellanic Cloud: the 280 s Be/X-ray binary pulsar AX J0058-7203 in outburst ${ }^{\star}$
}

\author{
F. Haberl and W. Pietsch
}

\begin{abstract}
Max-Planck-Institut für extraterrestrische Physik, Giessenbachstraße, 85748 Garching, Germany
e-mail: fwh;@mpe.mpg.de
\end{abstract}

Received 30 July 2007 / Accepted 27 September 2007

ABSTRACT

\begin{abstract}
Context. The Be/X-ray binary pulsar AX J0058-720, discovered by ASCA, was in the field of view of the EPIC instruments during five XMM-Newton observations in the direction of the Small Magellanic Cloud (SMC).

Aims. We used the EPIC data of the X-ray pulsar in order to investigate its long-term temporal and spectral behaviour between 2000 and 2005.

Methods. X-ray spectra covering $0.3-10 \mathrm{keV}$ and light curves in different energy bands were extracted from the EPIC PN and MOS data.

Results. The X-ray spectra are well modelled by a simple absorbed power-law without significant contribution from a soft component. The photon index of 0.7 indicates a relatively hard spectrum. During two observations in November 2005, separated by two days, the $0.2-10 \mathrm{keV}$ luminosity reached a maximum of $2.6 \times 10^{36} \mathrm{erg} \mathrm{s}^{-1}$ indicating an X-ray outburst. Twelve days later the source intensity had decreased by more than a factor of two. During an observation in November 2001 the source was not detected with a $2 \sigma$ upper limit of $2.8 \times 10^{33} \mathrm{erg} \mathrm{s}^{-1}$. Timing analysis of the observations of AX J0058-720 in 2005 reveals a pulse period of $286.3388 \pm 0.0012 \mathrm{~s}$ (or $286.8029 \pm 0.0012 \mathrm{~s}$ due to cycle count ambiguity). The average spin-down of $\sim 0.75 \mathrm{~s}$ per year between 1997 and 2005 suggests a moderate activity episode with low average accretion rate, insufficient to spin-up the neutron star.

Conclusions. The X-ray properties of AXJ0058-720 are typical for a Be/X-ray binary in the SMC. The source shows transient behaviour with more than a factor 900 intensity increases during outburst.
\end{abstract}

Key words. galaxies: individual: small Magellanic Cloud - galaxies: stellar content - stars: emission-line, Be - stars: neutron X-rays: binaries

\section{Introduction}

The discovery of X-ray pulsations from AX J0058-720 was reported by Yokogawa \& Koyama (1998) and a detailed temporal and spectral analysis of the ASCA data (Tsujimoto et al. 1999) revealed X-ray properties typical for a high mass X-ray binary of Be type. The pulsar exhibited coherent pulsations with a period of $280.3 \pm 0.3 \mathrm{~s}$ and a double peaked pulse profile. The X-ray spectrum was well modeled by a simple power-law with photon index of about 0.7 indicating a relatively hard spectrum. During the ASCA observation in 1997, Nov. 14-15 the measured flux in the $0.7-10.0 \mathrm{keV}$ band was $\sim 3 \times 10^{-12} \mathrm{erg} \mathrm{cm}^{-2} \mathrm{~s}^{-1}$ yielding a source luminosity of $\sim 1.4 \times 10^{36} \mathrm{erg} \mathrm{s}^{-1}$, assuming a distance of $60 \mathrm{kpc}$. From archival Einstein and ROSAT observations Tsujimoto et al. (1999) found the source highly variable in flux (by a factor of more than 10). The Be/X-ray binary nature was further strengthened by the tentative identification with the $\mathrm{H} \alpha$ emission line object 1036 in Meyssonnier \& Azzopardi (1993) by Haberl \& Sasaki (2000). This was finally confirmed by a refined X-ray position obtained by XMM-Newton (Sasaki et al. 2003) and by $\mathrm{H} \alpha$ spectroscopy (Coe et al. 2005). The XMM-Newton observation about three years after the ASCA observation revealed a pulse period increase to $281.1 \pm 0.2$ s (Sasaki et al. 2003). Schmidtke et al. (2006) present the

* Based on observations with XMM-Newton, an ESA Science Mission with instruments and contributions directly funded by ESA Member states and the USA (NASA) long-term OGLE-III light curve of AX J0058-720 which shows that the optical counterpart undergoes repeated outbursts with a period of $127.3 \pm 1.0$ days, which is most likely the orbital period of the binary system.

The X-ray pulsar AX J0058-720 is one of the well established Be/X-ray binaries in the Small Magellanic Cloud (SMC). With more than 60 such systems (including candidates in Haberl $\&$ Pietsch 2004) from which almost 50 have X-ray pulsations detected (Coe et al. 2005), the SMC is the ideal nearby galaxy to study a large population of these systems in a homogeneous way.

Apart from the first XMM-Newton observation of AX J0058-720 performed in 2000, Oct. 17 (Sasaki et al. 2003) the pulsar was in the field of view (FOV) of the EPIC instruments in four more pointings towards the SMC (centered on the massive binary star system HD 5980 as target). While in one of the observations the source was not detected, it was bright during two observations performed within two days in November 2005 indicating an outburst to a similar X-ray intensity as during the ASCA observation. We present the results of a temporal and spectral analysis of the EPIC data, the highest statistical quality X-ray data available so far from this Be/X-ray binary pulsar.

\section{Observations and data reduction}

Between Oct. 2000 and Dec. 2005 the Be/X-ray binary AX J0058-720 was in the EPIC field of view of five XMMNewton observations (at an off-axis angle of about $10^{\prime}$ ) in the 
Table 1. XMM-Newton EPIC observations of AX J0058-720.

\begin{tabular}{|c|c|c|c|c|c|c|c|c|}
\hline \multirow{4}{*}{$\begin{array}{c}\text { Observation } \\
\text { ID }\end{array}$} & $\begin{array}{l}\text { Pointin } \\
\text { RA } \\
\text { (J2 }\end{array}$ & $\begin{array}{l}\text { direction } \\
\text { Dec } \\
00.0)\end{array}$ & \multirow{2}{*}{$\begin{array}{l}\text { Sat. } \\
\text { Rev. } \\
0157\end{array}$} & $\begin{array}{c}\text { EPIC }^{1} \\
\text { Instrument } \\
\text { configuration }\end{array}$ & Start time (UT) & End time (UT) & $\begin{array}{l}\text { Duration } \\
\text { ksec }\end{array}$ & $\begin{array}{l}\text { Count } \\
\text { rate } \\
\text { cts s }^{-1}\end{array}$ \\
\hline & 005926.0 & -721011.0 & & PN EF & 2000-10-17 16:16:36 & 2000-10-17 20:41:09 & 15.9 & 0.16 \\
\hline & & & & M1 FF & 2000-10-17 15:10:44 & $2000-10-17$ 20:39:43 & 19.7 & 0.07 \\
\hline & & & & M2 FF & 2000-10-17 15:10:35 & $2000-10-17$ 20:39:42 & 19.7 & 0.06 \\
\hline \multirow[t]{3}{*}{0018540101} & 005926.8 & -720954.8 & 0357 & PN FF & 2001-11-21 00:21:48 & 2001-11-21 07:08:37 & 24.4 & - \\
\hline & & & & M1 FF & 2001-11-20 23:42:37 & 2001-11-21 07:13:02 & 27.0 & - \\
\hline & & & & M2 FF & $2001-11-2023: 42: 37$ & 2001-11-21 07:13:03 & 27.0 & - \\
\hline \multirow[t]{3}{*}{0304250401} & 005926.8 & -720954.0 & 1093 & PN FF & 2005-11-27 06:45:04 & 2005-11-27 11:10:41 & 15.9 & 0.79 \\
\hline & & & & M1 FF & 2005-11-27 06:22:46 & 2005-11-27 11:15:36 & 17.6 & 0.27 \\
\hline & & & & M2 FF & 2005-11-27 06:22:48 & 2005-11-27 11:15:41 & 17.6 & 0.25 \\
\hline \multirow[t]{3}{*}{0304250501} & 005926.8 & -720954.0 & 1094 & PN FF & 2005-11-29 05:33:52 & 2005-11-29 09:42:48 & 14.9 & 0.80 \\
\hline & & & & M1 FF & 2005-11-29 05:11:36 & 2005-11-29 09:47:43 & 16.6 & 0.27 \\
\hline & & & & M2 FF & $2005-11-2905: 11: 33$ & 2005-11-29 09:47:50 & 16.6 & 0.27 \\
\hline \multirow[t]{3}{*}{0304250601} & 005926.8 & -720954.0 & 1100 & PN FF & $2005-12-11$ 14:25:31 & 2005-12-11 17:21:37 & 10.6 & 0.20 \\
\hline & & & & M1 FF & 2005-12-11 12:48:45 & $2005-12-11 \quad 17: 26: 31$ & 16.7 & 0.12 \\
\hline & & & & M2 FF & 2005-12-11 12:48:42 & 2005-12-11 17:26:36 & 16.7 & 0.11 \\
\hline
\end{tabular}

${ }^{1} \mathrm{FF}$ : full frame CCD readout mode with $73 \mathrm{~ms}$ frame time; EF: extended full frame mode, $199 \mathrm{~ms}$; during all observations the medium optical blocking filter was used.

direction of the SMC which are summarized in Table 1. The EPIC cameras were operated in full frame imaging mode (during one observation PN was in extended full frame mode with longer CCD frame integration time) with medium filter. None of the observations was affected by strong background flares and we used the full exposure time for our analysis.

We extracted the data from the public XMM-Newton archive. We also searched through the Chandra archive, however no observation covered AX J0058-720 so far. For the XMMNewton data analysis we used SAS version 7.0.0 supported by tools from the FTOOL package together with XSPEC version 11.3.2p for spectral modelling.

\section{Results}

We obtained net source count rates $(0.2-4.5 \mathrm{keV})$ for AX J0058-720 using the maximum likelihood technique in SAS and list them in Table 1. A Fourier analysis of the EPIC data from Nov. 2005 clearly revealed the presence of the $280 \mathrm{~s}$ pulsations in the X-ray flux. As an example a power spectrum from Nov. 27 is presented in Fig. 1. The weaker of two prominent peaks is at the fundamental frequency while the stronger one represents the first harmonic of a double-peaked pulse profile. Using a folding technique we determine pulse periods to $286.56 \pm 0.04 \mathrm{~s}$, $286.41 \pm 0.04 \mathrm{~s}$ and $286.34 \pm 0.13 \mathrm{~s}$ ( $1 \sigma$ errors) for 2005 Nov. 27, 29 and Dec. 11, respectively. The pulse period history of AX J0058-720 including the ASCA and previous XMM-Newton data points is plotted in Fig. 2. Although there is some indication for spin-up during the last three XMM-Newton observations, the values are consistent within their $2 \sigma$ statistical uncertainties. Merging the data of the first two days and assuming a constant pulse period results in two equally probable periods of $286.322 \pm 0.002 \mathrm{~s}$ and $286.820 \pm 0.002 \mathrm{~s}$ due to the ambiguity of one spin cycle over the two day gap between the observations. Adding the Dec. 11 data does not allow to remove this ambiguity due to the lower source intensity and the too long gap of 12 days, resulting in periods of $286.3388 \pm 0.0012 \mathrm{~s}$ and $286.8029 \pm 0.0012 \mathrm{~s}$ with the lower value only marginally preferred. While the longer period is inconsistent with the periods determined from the individual observations the shorter period is

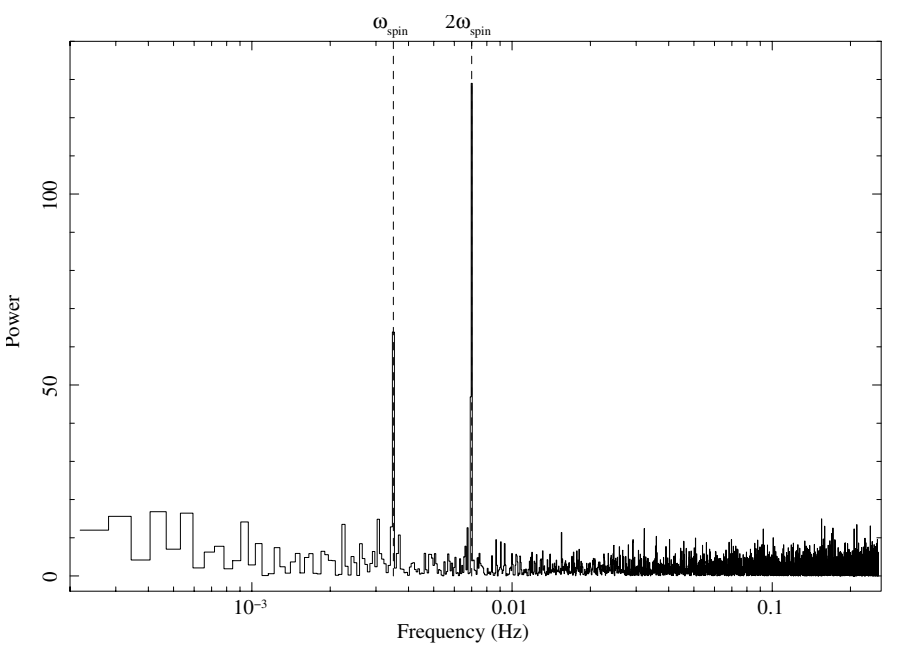

Fig. 1. Power spectrum of AXJ0058-720 obtained from the 0.5-10.0 keV EPIC-PN data of Nov. 27, 2005. The spin frequency and its first harmonic are indicated.

compatible with their "average" period. The fact that the value of $286.3388 \mathrm{~s}$ is more than $5 \sigma$ different from the value of the first observation again indicates spin-up (as it would be expected during a period of enhanced mass transfer during an outburst).

We folded the X-ray light curves in different energy bands as shown in Fig. 3, merging the data from the three observations end of 2005. The pulse profiles show two broad maxima separated $\sim 0.5$ in phase. The pulsed fraction decreases at energies above $4.5 \mathrm{keV}$ where the weaker peak nearly disappears.

We extracted EPIC spectra for PN (single + double pixel events, PATTERN 0-4) and MOS (PATTERN 0-12) excluding bad CCD pixels and columns (FLAG 0). Since the source was serendipitously observed at large off-axis angles, in most observations the source extraction regions are crossed by bad columns at various distances from the region center. Although the lost area in the extraction region is corrected for, the comparison of inferred fluxes from the three EPIC spectra shows, that the fluxes are generally underestimated when bad columns cross the extraction region. Fortunately, in all observations at least one 
Table 2. Results from absorbed power-law fits to the EPIC spectra.

\begin{tabular}{lcccccc}
\hline \hline Date & $2000-10-17$ & $2001-11-21$ & $2005-11-27$ & $2005-11-29$ & $2005-12-11$ & 2005 combined \\
Modified Julian Day & 51834.75 & 52234.16 & 53701.37 & 53703.32 & 53715.66 & - \\
Orbital phase ${ }^{1}$ & 0.17 & 0.31 & 0.84 & 0.85 & 0.95 & - \\
SMC $N_{\mathrm{H}}\left(10^{20} \mathrm{~cm}^{-2}\right)$ & $<6.2$ & - & $3.9_{-3.7}^{+3.9}$ & $4.8_{-4.4}^{+4.9}$ & $3.0_{-3.0}^{+15}$ & $3.7_{-2.1}^{+2.3}$ \\
Photon index & $0.76 \pm 0.08$ & - & $0.68 \pm 0.04$ & $0.72 \pm 0.05$ & $0.75 \pm 0.11$ & $0.70 \pm 0.02$ \\
$\chi_{r}^{2} /$ degrees of freedom & $0.99 / 43$ & - & $1.37 / 127$ & $1.07 / 120$ & $0.97 / 29$ & $1.23 / 280$ \\
Flux $\left(10^{-12} \mathrm{erg} \mathrm{cm}^{-2} \mathrm{~s}^{-1}\right)^{2}$ & $1.3(\mathrm{M} 2)$ & - & $5.9(\mathrm{M} 1)$ & $5.5(\mathrm{M} 1)$ & $2.2(\mathrm{M} 1)$ & - \\
Luminosity $\left(10^{36} \mathrm{erg} \mathrm{s}^{-1}\right)$ & 0.57 & $<2.8 \times 10^{-3}$ & 2.6 & 2.4 & 0.96 & - \\
\hline
\end{tabular}

${ }^{1}$ Using an orbital period of 127.3 days and the ephemeris for maximum optical light of MJD 52194.7 (Schmidtke et al. 2006) as phase 0.

${ }^{2} \mathrm{X}$-ray fluxes and luminosities are given for energies $0.2-10.0 \mathrm{keV}$ and the instrument from which they were derived is indicated: EPIC-MOS1 (M1); EPIC-MOS2 (M2). Errors denote the $90 \%$ confidence level.

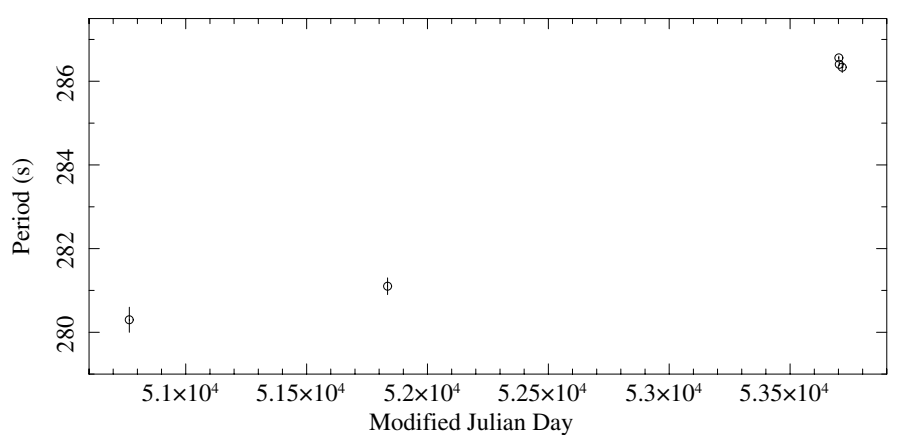

Fig. 2. Period history of AX J0058-720 from Nov. 1997 (discovery of pulsations with ASCA) to Nov./Dec. 2005.

of the three EPIC instruments allowed a complete source extraction and we give the flux obtained from that camera in Table 2.

The three EPIC spectra were simultaneously fit with the same model only allowing for a constant factor between the spectra to account for cross calibration problems $(<10 \%)$ but mainly for area losses in the extraction region (up to 30\%). As model a simple absorbed power-law was used with two absorption column densities accounting for the interstellar absorption in the Milky Way (using elemental abundances of Wilms et al. (2000)) and in the SMC (with metal abundances set to 0.2 as typical for the SMC; Russell \& Dopita 1992). The Galactic foreground $N_{\mathrm{H}}$ was fixed at $6 \times 10^{20} \mathrm{~cm}^{-2}$. The results of the fits are summarized in Table 2 . The derived column densities and photon indices for the individual observations agree within their errors and, therefore, we merged the spectra for each camera from the three observations performed in 2005. The merged 2005 EPIC spectra are shown in Fig. 4 together with the best fit model.

We note here that the flux derived from the observation in Oct. 2000 is considerably higher than the value given by Sasaki et al. (2003) (source 7 in their Table 2) based on the same data. This is due to the following corrections which were not applied in the older analysis based on version 5.3.3 of the SAS software: vignetting (the source is at a relatively large off-axis angle), point spread function losses outside the source extraction area and the problems with bad columns as described above. In addition we use a slightly wider energy band.

Assuming the model parameters derived from the merged EPIC spectra we convert the count rates of the ROSAT detections (from the source catalogues of pointed PSPC and HRI observations $2 \mathrm{RXP}$ and $1 \mathrm{RXH}$ ) into $0.2-10 \mathrm{keV} \mathrm{lu}-$ minosities: $\sim 2.0 \times 10^{35} \mathrm{erg} \mathrm{s}^{-1}$ for the PSPC detection of $3.6 \times 10^{-3} \mathrm{cts} \mathrm{s}^{-1}$ (observation 600195n00, 1991, Oct. 8 to Nov. 3) and $\sim 4.3 \times 10^{35} \mathrm{erg} \mathrm{s}^{-1}$ and $\sim 2.0 \times 10^{36} \mathrm{erg} \mathrm{s}^{-1}$ for the two HRI detections with $2.9 \times 10^{-3}$ cts s$^{-1}$ and $1.4 \times 10^{-2}$ cts s $^{-1}$ (observations 500418a03 and 600928n00 in 1998, March 27 to April 1 and 1997, Oct. 2 to Nov. 23. We note here that the conversion of ROSAT count rates in the soft $0.1-2.4 \mathrm{keV}$ energy band to luminosities strongly depends on the assumed absorption column density. However, all EPIC spectra indicate relatively low $N_{\mathrm{H}}$, only marginally above the Galactic foreground value and without significant variation with time. Therefore, using XMM-Newton spectra for the luminosity conversion of ROSAT count rates can be justified.

The X-ray source was not detected during the Nov. $2001 \mathrm{ob}-$ servation and we derived an upper limit of $7.9 \times 10^{-4} \mathrm{cts} \mathrm{s}^{-1}(2 \sigma$, $0.5-4.5 \mathrm{keV})$. Assuming the spectral parameters obtained from the 2005, Nov. 27 EPIC spectra this corresponds to a luminosity upper limit of $2.8 \times 10^{33} \mathrm{erg} \mathrm{s}^{-1}(0.2-10 \mathrm{keV})$ which is given in Table 2. This is the lowest upper limit ever determined for AX J0058-720 resulting in a ratio between observed maximum and minimum flux of more then 930 .

\section{Discussion}

We present results from a temporal and spectral analysis of XMM-Newton EPIC data of the Be/X-ray pulsar AX J0058-720 located in the SMC. Since the discovery of AX J0058-720 by ASCA (1997, Nov. 14-15) the pulse period has increased from $280.4 \pm 0.3 \mathrm{~s}$ (Tsujimoto et al. 1999) to $286.3388 \pm 0.0012 \mathrm{~s}$ (or $286.8029 \pm 0.0012 \mathrm{~s}$ due to cycle count ambiguity) during the XMM-Newton observations in Nov./Dec. 2005. This corresponds to an average spin-down of $\sim 0.75 \mathrm{~s} \mathrm{yr}^{-1}$. However, the first XMM-Newton observation three years after the ASCA observation indicated a relatively slow spin-down $\left(\sim 0.23 \mathrm{~s} \mathrm{yr}^{-1}\right)$ and most of the period change occurred between the years 2000 and $2005\left(\sim 1.05 \mathrm{~s} \mathrm{yr}^{-1}\right)$. Opposite to this period increase, other $\mathrm{Be} / \mathrm{X}$-ray binary pulsars in the SMC with similar spin periods like e.g. SAX J0103.2-7209 and AX J0051-733 (Haberl \& Pietsch 2004) show a period decrease, i.e. spin-up. The episode of spin-down, together with the non-detection of the source in 2001 indicates that AX J0058-720 is so far observed in a low activity epoch with low long-term average accretion rate. The X-ray luminosity during the Nov. 2005 observations was again at about the same level as during the ASCA observation in Nov. 1997 and the ROSAT observation in Oct./Nov. 1997, suggesting typical outburst maxima of AX J0058-720 close to $2-3 \times 10^{36} \mathrm{erg} \mathrm{s}^{-1}$. Such relatively low outburst maxima make it also understandable that the RXTE monitoring of X-ray pulsars in the SMC did not yield any detection of AX J0058-720 (the RXTE detection limit for pulsations in the SMC monitoring is $\sim 10^{36} \mathrm{erg} \mathrm{s}^{-1}$ on-axis (see Laycock et al. 2005).

Although the short XMM-Newton observations do not allow us to determine the times of the maxima of the X-ray outbursts, 


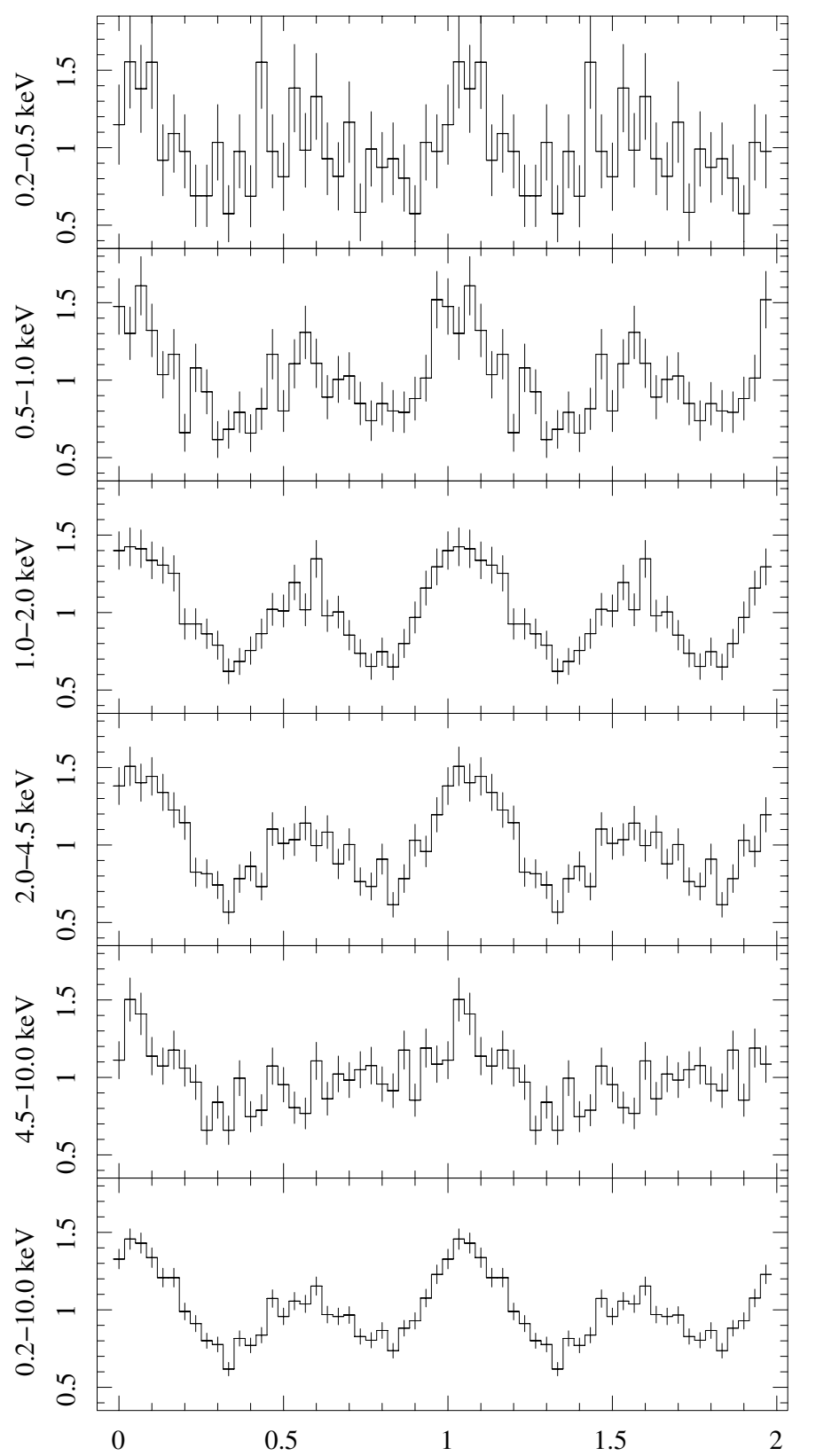

Phase (286.3388 s)

Fig. 3. Folded EPIC-PN light curves in the standard EPIC energy bands including the data from the three observations in Nov./Dec. 2005. The intensity profiles are normalized to the average count rate (in cts s ${ }^{-1}$ : $0.0126,0.0328,0.0686,0.0701,0.0559,0.241$ from top to bottom) and include background (average levels of 0.0021, 0.0013, 0.0013, 0.0014, $\left.0.0034,0.0097 \mathrm{cts} \mathrm{s}^{-1}\right)$.

the covered phases (Table 2) calculated from the ephemeris and optical period given by Schmidtke et al. (2006) indicate a correlation of X-ray and optical outbursts: The four XMMNewton observations in which the source was detected all cover phases during the relatively broad optical outburst (see Fig. 4 in Schmidtke et al. 2006) while the non-detection in Nov. 2001 was

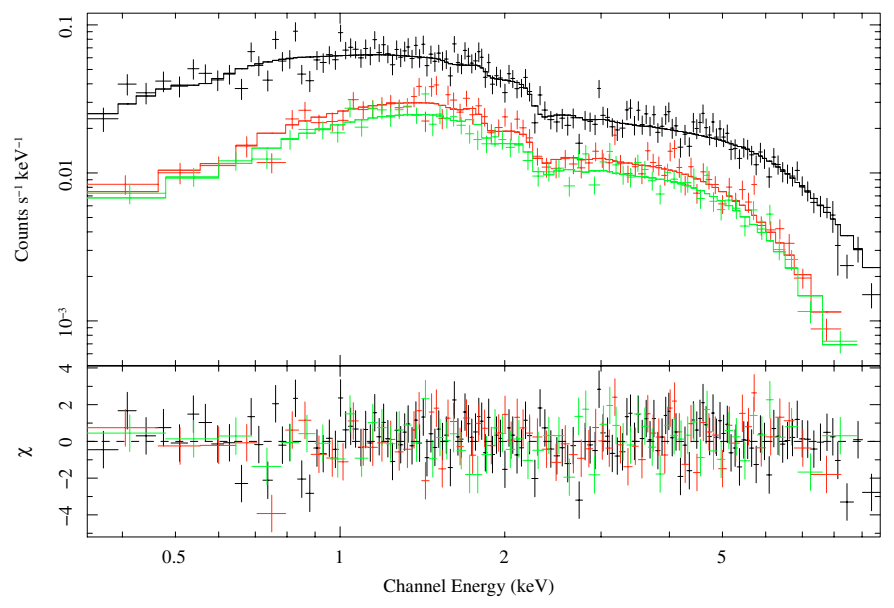

Fig. 4. Merged Nov./Dec. 2005 EPIC spectra of AX J0058-720. EPICPN is shown in black and EPIC-MOS in red (M1) and green (M2) (both grey in black and white representation). The histograms show the bestfit absorbed power-law model.

after the optical outburst. This supports the interpretation of the optical outburst period as orbital period of the binary system.

The X-ray spectrum of AX J0058-720 in the $0.2-10 \mathrm{keV}$ band can be well represented by an absorbed power-law. Although the absorbing column density towards the source is low no significant soft component is detected as it is seen from other Be/X-ray binaries in the SMC (see the discussion in Haberl \& Pietsch 2005). The photon index of 0.7 inferred from the EPIC spectra is consistent with the value found from the ASCA spectrum and indicates a spectrum slightly harder than on average seen from Be/X-ray binaries in the SMC (Haberl $\&$ Pietsch 2004). The spectral slope does not vary significantly while the X-ray luminosity changes by a factor of five. Also we find no indication for changes in the absorption column density. Overall, the X-ray properties of AX J0058-720 are typical for a moderately active $\mathrm{Be} / \mathrm{X}$-ray binary with transient behaviour.

Acknowledgements. The XMM-Newton project is supported by the Bundesministerium für Wirtschaft und Technologie/Deutsches Zentrum für Luft- und Raumfahrt (BMWI/DLR, FKZ 50 OX 0001), the Max-Planck Society and the Heidenhain-Stiftung.

\section{References}

Coe, M. J., Edge, W. R. T., Galache, J. L., \& McBride, V. A. 2005, MNRAS, 356,502

Haberl, F., \& Pietsch, W. 2004, A\&A, 414, 667

Haberl, F., \& Pietsch, W. 2005, A\&A, 438, 211

Haberl, F., \& Sasaki, M. 2000, A\&A, 359, 573

Laycock, S., Corbet, R. H. D., Coe, M. J., et al. 2005, ApJS, 161, 96

Meyssonnier, N., \& Azzopardi, M. 1993, A\&AS, 102, 451

Russell, S. C., \& Dopita, M. A. 1992, ApJ, 384, 508

Sasaki, M., Pietsch, W., \& Haberl, F. 2003, A\&A, 403, 901

Schmidtke, P. C., Cowley, A. P., \& Udalski, A. 2006, AJ, 132, 971

Tsujimoto, M., Imanishi, K., Yokogawa, J., \& Koyama, K. 1999, PASJ, 51, L21

Wilms, J., Allen, A., \& McCray, R. 2000, ApJ, 542, 914

Yokogawa, J., \& Koyama, K. 1998, IAU Circ., 6853, 2 